\title{
Formas de organización de las Experiencias de Lectura Colectiva en Clases de comprensión de textos ${ }^{1}$
}

\section{Formas de organizar as Experiências de Leitura Coletiva nas Aulas de compreensão de textos}

\section{Methods to organize Collective Reading Experiences in Language Classes on Text Comprehension}

\author{
Carolina Iturra Herrera ${ }^{2}$
}

\begin{abstract}
RESUMEN
El presente estudio buscó describir la manera en que se organizaba el trabajo conjunto de profesores y estudiantes, en el aprendizaje de la comprensión de textos. Para ello, se seleccionaron 30 sesiones del Sistema Nacional de Evaluación del Desempeño Docente año 2008, en lenguaje y comunicación. Mediante la segmentación del discurso, se analizó cada sesión identificando las tareas desplegadas y la secuenciación de estas a lo largo de la clase, considerando los momentos de la lectura: antes, durante y después. Los resultados evidencian que en todas las clases se identificaron tareas asociadas a los tres momentos, sin embargo, difirieron en la frecuencia y en la organización secuencial. Ello sugiere una variabilidad en las formas de enfrentar la enseñanza de la comprensión de textos.
\end{abstract}

Palabras clave: comprensión de textos; práctica educativa; enseñanza de la lectura.

DOI: $10.1590 / 0104-4060.37089$

1 El estudio fue realizado gracias a los fondos del proyecto Fondecyt (Fondo Nacional de Desarrollo Científico y Tecnológico) N. ${ }^{\circ} 11110158$. Se agradece también el apoyo del Centro de Estudios del Ministerio de Educación y de Centro de Perfeccionamiento, Experimentación e Investigaciones Pedagógicas - CPEIP, quienes dieron acceso al material incluido en el estudio.

2 Universidad de Talca. Facultad de Psicología. Talca, Séptima Región, Chile. Av. da Lircay s/n. Campus Lircay. Casilla 747-721. 


\title{
RESUMO
}

Este estudo teve como objetivo descrever a maneira que se organizou o trabalho conjunto de professores e alunos na aprendizagem da compreensão de textos. Para isso, foram selecionadas 30 sessões da Avaliação Nacional de Desempenho de Ensino de 2008, em linguagem e comunicação. Segmentando o discurso, cada sessão foi analisada por meio da identificação e sequenciação das tarefas desenvolvidas, no decorrer das aulas, considerando os momentos de leitura: antes, durante e depois. Os resultados mostram que em todas as aulas as tarefas foram associadas a esses três momentos, no entanto, elas diferem na frequência e na organização sequencial. Isto sugere uma variabilidade nas formas de lidar com o ensino da compreensão de textos.

Palavras-chave: compreensão de textos; prática educativa; ensino de leitura.

\begin{abstract}
This study describes the way teachers and students' joint work about text comprehension learning was organized. Therefore, 30 sessions on language and communication were selected from the Chilean National Teaching Performance Assessment, 2008. Using speech segmentation, each session was analyzed, identifying the deployed tasks and their sequencing along the classes, considering three phases: before, during and after reading. Results show that tasks were associated with those three moments in each analyzed class, although such tasks differed in frequency and sequence within each phase and class observed. This suggests variability among teachers in their ways of dealing with the teaching of reading comprehension.
\end{abstract}

Keywords: reading comprehension; educational practice; teaching of reading.

\section{Introducción}

En las últimas décadas ha surgido la necesidad de abordar el estudio y análisis de las acciones que emergen cuando profesores y alumnos trabajan conjuntamente en un aula. Para ello, se hace necesario describir cómo se organiza la enseñanza, mediante las distintas actividades, tareas y pasos que las componen (WELLS, 2001), conceptualizando estas actividades como las distintas acciones conjuntas que llevan a cabo profesores y alumnos en un aula. Esta estructuración dependería del tipo de contenido instruccional (STODOLSKY, 1991), dado que este delimitaría el formato instruccional desplegado por el profesor, el nivel cognitivo que persiguen las tareas y los modos de participación de profesores 
y estudiantes. Además, estas acciones estarían supeditadas al carácter dinámico de las interacciones, es decir, a la ordenación y jerarquización de las actividades y tareas, que consideraría la naturaleza propia de los contenidos escolares que se entrelazaría con el modo en que profesores y estudiantes enfrentan la tarea, entendiendo que sus acciones, situadas en el contexto del aula, determinarían en parte la ejecución de las mismas.

En otras palabras, una actividad se estructura y operacionaliza en la práctica, debido a que los objetivos son dinámicos y emergentes, y dependen de las negociaciones que efectúen los profesores y alumnos en la interacción y operacionalización de la actividad. De esta manera, aun cuando estas actividades pudieran ser intencionalmente planificadas por el enseñante, cobran vida y se concretan en el contexto de un aula, dependiendo de las distintas relaciones y expectativas mutuas entre los participantes de una interacción, los tipos de conductas verbales y no verbales que despliegan y los tipos de instrumentos semióticos que utilizan (discurso, textos).

Desde este punto de vista, Ratner (1997 apud DANIELS, 2003, p. 114) propone que:

La actividad cultural y los fenómenos sicológicos dependen y se sustentan mutuamente... Los fenómenos sicológicos son los procesos subjetivos de la actividad cultural práctica y la actividad cultural práctica es la vertiente práctica y objetivada de los fenómenos sicológicos que componen la vida social organizada.

De acuerdo a esta perspectiva, la propia organización de las actividades y tareas en función de la naturaleza de los contenidos escolares se entrelazaría con la manera en que los aprendices enfrentan la tarea, entendiendo que sus acciones, situadas en el contexto del aula, determinarían en parte la ejecución de las mismas.

En consecuencia, un factor primordial para entender el proceso de enseñanza-aprendizaje se vincularía directamente con la estructuración de las actividades y tareas, dado que éstas, además de regular en parte el microsistema denominado aula, organizarían las distintas acciones o rutas a seguir, obedeciendo la naturaleza propia de los contenidos escolares. Este modo de organizar las acciones repercutiría directamente en la forma cómo se aprenden los contenidos escolares. 


\section{La comprensión de textos en las aulas}

Para alcanzar la competencia lectora en las aulas es necesario un trabajo de colaboración entre profesor y alumno, orientado a generar estrategias que le permitan a este último alcanzar desempeños adecuados en este dominio. Por tanto, se entiende que la comprensión de textos es una actividad conjunta en el aula, que entrelaza las acciones de los profesores, estudiantes y al texto y sus particularidades. De este modo, durante la interacción en las aulas, se relaciona al texto y sus posibilidades interpretativas, al lector y las ayudas prestadas por el profesor.

$\mathrm{Al}$ respecto, se reconoce en la actualidad la necesidad de sustentar la instrucción en la comprensión de textos a partir del diálogo entre profesores y estudiantes, entendiendo que las aulas son espacios de interacción social y comunicacional (APPLEBEE et al., 2003; NYSTRAND, 2006). Estos diálogos entre profesores y estudiantes permitirían configurar las distintas acciones y actividades que enmarcarían la enseñanza de la comprensión de textos, orientados aquellos procesos que los estudiantes deben aprender para lograr una adecuada comprensión.

Así por ejemplo, Graves y Graves (2003; CLARK; GRAVES, 2005) señalan un marco instruccional denominado Scaffolded Reading Experience (SRE) que facilitaría el logro de la comprensión de textos en los estudiantes. Este marco se constituiría de dos componentes: la planificación y la implementación. En la planificación, los profesores organizarían técnicas, estrategias y recursos considerando las necesidades de los estudiantes, la dificultad del texto y los propósitos para la lectura, incorporando actividades docentes planificadas antes, durante, y después de leer, en tanto que la implementación se definiría como la inclusión en la acción de distintas tareas específicas que apoyarían el proceso comprensivo de los estudiantes. Desde esta perspectiva, los profesores deben planificar actividades en la fase de prelectura para establecer una meta y activar el conocimiento previo de los estudiantes sobre el tema, en la fase durante la lectura incorporar tareas que permitan monitorear la comprensión de los estudiantes, como el conocimiento de la estructura del texto. Finalmente, en la fase después de la lectura, la instrucción se orientaría a resumir, discutir sobre las ideas del texto o esquematizar lo leído. A partir de lo anterior, Liang, Peterson \& Graves (2005) investigaron los efectos de la instrucción en Scaffolded Reading Experience para fomentar la comprensión de la literatura en estudiantes de primaria. Para ello, diseñaron un estudio cuasi-experimental que investigó los efectos de dos enfoques para el fomento de la comprensión de la literatura en estudiantes de primaria, siendo uno de ellos el SRE. Dentro de los resultados obtenidos, plantearon que en las pruebas de opción múltiple, los estudiantes que 
recibieron SRE puntuaron significativamente más alto que los estudiantes que recibieron el otro enfoque. En este sentido, los autores plantearon la necesidad de estructurar las experiencias de lectura a partir de procedimientos didácticos que actúen como andamios del proceso comprensivo.

Por otro lado, Sánchez et al. (2008a; SÁNCHEZ; GARCÍA; ROSALES, 2010) han analizado la secuenciación y organización de actividades típicas de lectura colectiva, definidas como actividades regulares orientadas a la comprensión de un texto, que cuenta con un plan de trabajo reconocido por los participantes de la actividad (LEMKE, 1997). Como resultado de su trabajo, han podido identificar la secuencia temporal que ordena el conjunto de tareas que constituyen las actividades típicas de lectura, logrando caracterizar el modo en que se organiza y secuencia cada una y el papel juega cada uno de los componentes en dicha actividad. Al respecto, establecieron ciertos patrones precisando que los más característicos en las clases que han analizado son patrones simples que carecen de actividades orientadas a establecer metas de lectura y/o activar conocimientos previos, y que se caracterizarían principalmente por leer los textos y posteriormente interpretar o evaluar los mismos. Además de estos, existirían los patrones complejos que incluirían acciones orientadas a establecer metas de lectura, activar conocimientos previos, leer e interpretar/evaluar lo leído, confeccionar mapas o esquemas, etc. Por tanto, las diferencias entre un patrón y otro radicarían en la inclusión de un mayor número de actividades, pero además, en la conducción explícita del proceso, mediante una meta o propósito de la experiencia y la activación de conocimientos previos.

En síntesis, las propuestas revisadas recalcan la necesidad de que durante la interacción entre profesores y estudiantes orientadas al trabajo comprensivo se desplieguen e incorporen distintas actividades y tareas, que permitan apoyar el proceso comprensivo de los estudiantes.

\section{Objetivos}

El estudio de carácter descriptivo se orienta a analizar la interacción profesor-alumno en clases dedicadas a la comprensión de textos en segundo ciclo de educación básica. Se pretende de esta manera caracterizar las distintas configuraciones que articulan a cada una de estas clases, identificando el modo en que se construyen, organizan y secuencian las distintas tareas y actividades cuando profesores y estudiantes trabajan conjuntamente en la comprensión de un texto. 


\section{Método}

\section{Participantes}

Se seleccionaron 30 grabaciones de clases pertenecientes al sistema de evaluación del desempeño docente chileno del año 2008. Estas grabaciones consisten en sesiones de 40 minutos de clases, en las que participan los profesores evaluados y sus estudiantes, por tanto, se seleccionaron 30 profesores. Las grabaciones forman parte del proceso de evaluación del desempeño docente y corresponden al módulo 2 del instrumento de evaluación denominado portafolio (CHILE, 2004). Para seleccionarlas, se establecieron criterios muestrales intencionales (RUIZ, 2012; FLICK, 2012) orientados principalmente a que la sesiones incluyeron la comprensión de textos como contenido escolar y que provinieran del nivel escolar de segundo ciclo de primaria. Este ciclo educativo se organiza en función de 4 grados escolares, $5 .^{\circ}, 6 .^{\circ}, 7 .^{\circ}$ y $8 .^{\circ}$ y las edades de los estudiantes oscilan entre los 10-14 años.

Además de ello, se accedió a todo el material contenido en la planificación de la sesión grabada y a los niveles de desempeño docente obtenidos por los profesores, tal y como se muestra en la Tabla 1.

TABLA 1 - CARACTERIZACIÓN DE LA MUESTRA

\begin{tabular}{llllll}
\hline & \multicolumn{5}{c}{ Nivel de enseñanza (grados) } \\
\cline { 2 - 6 } Evaluación desempeño docente & 5 grado & 6 grado & 7 grado & 8 grado & total \\
\hline Básico & 6 & 3 & 6 & 1 & 15 \\
Competente & 5 & 3 & 5 & & 13 \\
Destacado & 1 & & 1 & & 2 \\
Total & 11 & 6 & 12 & 1 & 30 \\
\hline
\end{tabular}

\section{Sistema de análisis}

Para analizar la interacción profesor-alumno, se empleó el sistema de análisis propuesto por Sánchez, García \& Rosales (2010), quienes han diseñado un modelo orientado a estudiar tareas específicas de comprensión de textos y que cuenta un procedimiento declarado (SÁNCHEZ et al., 2008b) que define distintas unidades de análisis que permiten ir desfragmentando las interacciones que se dan en las aulas, para de esta manera caracterizar las organizaciones 
globales o locales del proceso de enseñanza-aprendizaje de la comprensión. La Cuadro 1 ofrece un resumen de las distintas unidades de análisis propuestas por estos autores.

\begin{tabular}{|l|l|}
\hline Unidades de análisis & Definición \\
\hline Sesión, clase o lección & $\begin{array}{l}\text { Cada una de las unidades temporales en las que se desarrolla } \\
\text { una Unidad Curricular. }\end{array}$ \\
\hline $\begin{array}{l}\text { Actividad típica de } \\
\text { aula (ATA) }\end{array}$ & $\begin{array}{l}\text { Actividades regulares con un objetivo y un plan de traba- } \\
\text { jo conocido que conforman cada una de las sesiones y que } \\
\text { se repite habitualmente a lo largo de una UC. Por ejemplo: } \\
\text { producción de textos, explicaciones verbales, desarrollo de } \\
\text { tareas, etc. (LEMKE, 1997). }\end{array}$ \\
\hline Episodio & $\begin{array}{l}\text { Si la ATA es muy compleja, cabe diferenciar varias metas di- } \\
\text { ferentes que suelen ser necesarias para su desarrollo. El de- } \\
\text { sarrollo de cada una de esas metas principales para el logro } \\
\text { del objetivo de la actividad típica constituiría un episodio, } \\
\text { que suele tener una estructura de participación reconocible. }\end{array}$ \\
\hline Ciclo & $\begin{array}{l}\text { Conjunto de intercambios necesarios para que dos o más } \\
\text { personas lleguen a un acuerdo o, al menos, crean haber lle- } \\
\text { gado a un acuerdo respecto del desarrollo de alguna meta (o } \\
\text { submeta). }\end{array}$ \\
\hline
\end{tabular}

CUADRO 1 - UNIDADES DE ANÁLISIS

En concreto, para esta investigación se utilizaron dos unidades de análisis. La primera denominadas Actividades Típicas (ATAs), que permiten segmentar la interacción discursiva entre los participantes, considerando el conjunto de acciones o tareas con un propósito definido, que en este caso sería la comprensión de un texto. Al respecto, es necesario señalar que en una unidad didáctica o sesión de clases podría identificarse distintas ATAs, tales como la explicación del profesor, en que el foco de la interacción estaría dado por la explicación del profesor de una materia o temática determinada, lo que supondría ciertas estructuras de participación.

La segunda unidad de análisis utilizada fue de los episodios, que operan a un nivel más local, posibilitando la segmentación de las actividades típicas de lectura en las distintas tareas o pasos requeridos para alcanzar el propósito establecido en la actividad típica. En este sentido, la investigación en torno a las actividades típicas de lectura colectiva ha identificado episodios de planificación, conocimientos previos, interpretación, evaluación, mapa conceptual, entre otros (SÁNCHEZ; GARCÍA; ROSALES, 2010). 


\section{Procedimiento y análisis de datos}

Una vez seleccionada la muestra, se procedió a la transcripción literal de las sesiones recogiendo los distintos turnos de habla de profesores y estudiantes, logrando así contar con 30 sesiones transcritas. Posteriormente, se analizaron los turnos de habla de cada transcripción, con el objeto de identificar las ATAs que componían a cada sesión. Para ello, se analizó el discurso y la interacción verbal entre los participantes, segmentando aquellos que estaban dirigidos a alcanzar un propósito común, que en este caso era comprender un texto. De esta manera, se aislaron todas las acciones enmarcadas por este propósito central. El resto de las ATAs identificadas no fueron analizadas.

En segundo lugar, una vez identificada cada actividad típica de lectura colectiva, se procedió a descomponerlas en los episodios que las constituyeron. Para ello, se utilizaron como criterios de división dos aspectos. El primero vinculado a la identificación de la meta u objetivo específico de cada conjunto de interacciones verbales necesario para el logro del propósito principal; y el segundo relacionado al reparto de roles característicos en cada uno.

Una vez segmentados e identificados los distintos tipos de episodios, se confeccionaron Mapas de Actividades típicas (ATA) que permitieran representar temporalmente la organización secuencial de cada una de las Actividades típicas analizadas. Estos Mapas de Actividades típicas se ordenaron en función de las distintas fases del ciclo de lectura (antes, durante y después).

Como criterio de veracidad (GUBA, 1981) se utilizó para la credibilidad las grabaciones de las clases en formato de audio y video, además de la transcripción literal de las mismas, lo que permitió acceder las veces necesarias a la fuente de datos. La transferibilidad se logró mediante la descripción de las características de la muestra y los criterios para su selección. Finalmente, para la dependencia, se empleó la triangulación de criterios de segmentación, participando en este procedimiento codificadores capacitados en el sistema de análisis.

\section{Resultados}

Se lograron identificar un total de 30 actividades típicas de lectura colectiva, correspondiendo una a cada una de las sesiones seleccionadas. Por otro lado, se identificaron un total de 425 episodios en las 30 actividades típicas de 
lectura colectiva, agrupándolos en 22 tipos de episodios distintos. Cada uno se caracterizó por contar con una meta distintiva, es decir, desarrollar una tarea o acción dirigida a lograr el propósito de la ATA.

En relación a los tipos de episodios o tareas más frecuentemente desplegadas en cada una de las experiencias de lectura colectiva analizadas fueron planificación, conocimientos previos, lectura, recapitulación, interpretación de las ideas del texto, instrucciones de guía de trabajo, desarrollo de guía de trabajo y revisión de guía de trabajo. Mientras que los de menor frecuencia fueron repaso, mapa conceptual, identificación de noticias y elaboración conjunta de la guía de trabajo. Además de estas diferencias, cada sesión se organizó secuencialmente de forma particular que pasaremos a revisar a continuación.

\section{Momentos de Lectura}

En términos generales, la organización lograda en cada una de las actividades típicas analizadas permitió detectar en todas ellas los tres momentos, algunos de ellos más homogéneos que otros. A continuación se presentarán los resultados obtenidos en cada uno de estos momentos.

\section{Antes de la lectura: la preparación del proceso}

La totalidad de las actividades típicas analizadas desplegaron episodios dirigidos a establecer la meta de lectura, así como activar conocimientos previos. La excepción se detectó en la ATA n..$^{\circ}$, en la que no desplegó un episodio dirigido a activar conocimientos previos, tal y como se muestra en la Figura 1.

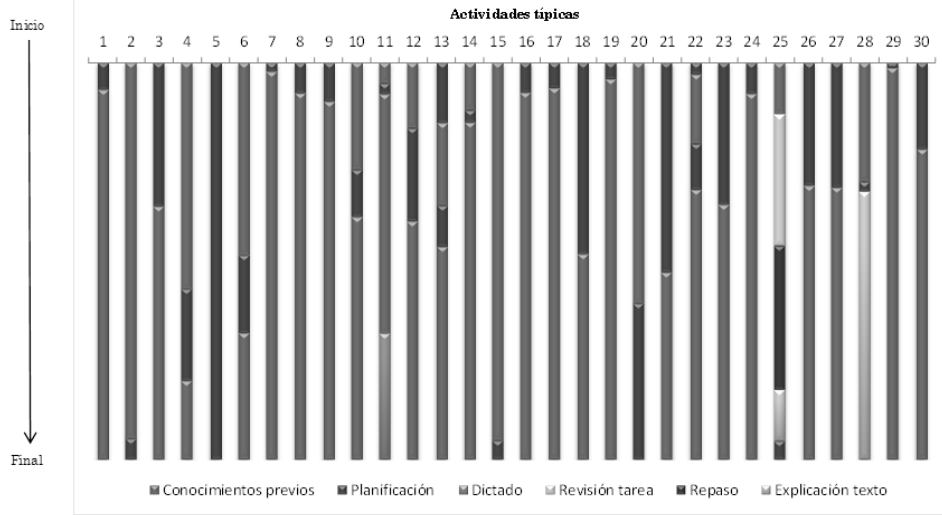

FIGURA 1 - MAPA DE EPISODIOS MOMENTO ANTES DE LA LECTURA 
La figura también permite observar que, en la mayoría de las ATAs, los episodios dedicados a dar inicio a la actividad de lectura siguieron una secuencia de episodio de planificación para continuar con un episodio de conocimientos previos, no obstante, en algunos casos esta organización se invirtió dando inicio a la actividad a través de la activación de conocimientos previos para luego explicitar la meta y objetivo de la clase (ATAs 2 y 20). Adicionalmente, en un porcentaje de ellos se agregaron, a posteriori del episodio de planificación, otro episodio de conocimientos previos o de otras características. En estas situaciones, las estrategias desplegadas por los profesores fueron primero generar un contexto cognitivo activando conocimientos relacionados con el tipo de texto a trabajar o evocaciones suscitadas con recursos didácticos como dibujos o imágenes, entre otras, para luego explicitar el objetivo de la clase y retomar los conocimientos vinculados directamente al texto objeto de lectura.

Por ejemplo, la ATA 11 inició con un episodio de conocimientos previos relacionados a las leyendas, posteriormente, la profesora explicitó el objetivo de la clase, retomando la activación de conocimientos previos, mediante la utilización de las preguntas contenidas en los libros de texto y finalizando este momento con un episodio de dictado relacionado con el concepto de leyenda.

Un caso particular es la secuencia seguida por la ATA 25, que inició la actividad con preguntas iniciales dirigidas a recordar lo trabajado en la sesión anterior, para después revisar una tarea relacionada al contenido trabajado. Posteriormente, se repasó conjuntamente el concepto de leyenda, volviéndose a indagar en los alumnos sus ideas previas, pero esta vez, utilizando un recurso didáctico vinculado a una característica del texto que leerán en la sesión. Esta fase terminó con la explicitación del objetivo de la lectura "Ya, el objetivo de la clase de hoy es leer, comprender, analizar y reflexionar, en torno a la leyenda urbana."

En resumen, durante la preparación a la lectura, la mayoría de las actividades típicas iniciaron esta fase con la explicitación del objetivo de la clase, para desde ahí, abordar la activación de conocimientos previos mediante indagaciones. Sin embargo, se observaron casos en los que la secuencia se invirtió u otros casos en los que se incluyeron tareas distintas.

\section{Durante la lectura: la supervisión de la comprensión}

Uno de los propósitos de esta fase, además de la lectura del texto, es la utilización de diversas estrategias y recursos que permitan monitorear el sentido del texto y que faciliten la comprensión del mismo.

Al respecto, una parte importante de las ATAs comenzó la fase con un episodio dedicado a entregar instrucciones orientadas principalmente a organizar 
los turnos de lectura. Además de esto, se identificaron dos formas distintas de organizar esta fase. La primera, presente en 15 actividades típicas analizadas, fue simplemente leer el texto en voz alta, acción que mayoritariamente era responsabilidad de los estudiantes. El segundo modo, identificado en las 15 ATAs restantes, consistió en intercalar fragmentos de lectura con otro tipo de acción, como intercambios comunicativos orientados a supervisar el sentido de la lectura, clarificar vocabulario o entregar instrucciones para realizar las guías de trabajo en el aula, tal y como se recoge en el Mapa de ATAs de esta fase (Figura 2).

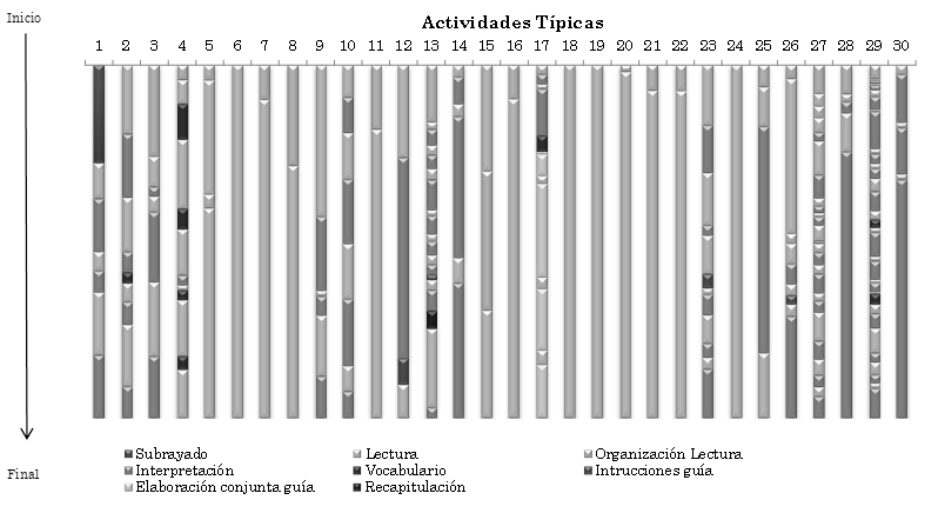

FIGURA 2 - MAPA DE EPISODIOS MOMENTO DURANTE DE LA LECTURA

De estas acciones, la de mayor frecuencia fue el intercalar un episodio o tarea de lectura con uno de interpretación. Este movimiento implicaba, por tanto, organizar las acciones en "Leemos un fragmento del texto y después revisamos lo leído." La diferencia entre aquellas ATAs en las que se detectó esta configuración u organización fue del número de episodios intercalados, como por ejemplo, la ATA 25 en que sólo se contabilizó una acción de esta naturaleza, versus la ATA 29, en la que fue posible identificar 14 de movimientos que involucraban intercalar la lectura con la interpretación de la misma.

La segunda configuración denominada lectura-vocabularios (ATAs 4 y 29) se articuló de un episodio de lectura seguido de uno de revisión de vocabulario, representando ello la acción de "leemos un fragmento del texto y revisamos el significados de algunas palabras extraídas de la lectura".

Por último, la tercera configuración combinaba un episodio de lectura y uno de elaboración conjunta de guía (ATA 17). En este caso, la profesora, después de leer el texto con los estudiantes en voz alta, focalizó el trabajo conjunto en ir respondiendo las preguntas contenidas en la guía de trabajo, recurso didáctico 
elaborado por los profesores para ir supervisando la comprensión de textos. En este sentido, la acción comenzó a desarrollarse a partir de las instrucciones de la guía, para después proseguir con la lectura del texto, seguido de indagaciones dirigidas a responder conjuntamente y en voz alta, algunas preguntas de la guía, para retomar nuevamente la lectura y continuar con las indagaciones.

En resumen, aun cuando en todas las ATAs se identificó esta fase, cerca del $50 \%$ de ellas (15 ATAs) organizó esta fase a partir de la inclusión de acciones de supervisión, de las cuales la más recurrente fue el intercalar la lectura con otras tareas o episodios.

\section{Después de la lectura: la profundización de lo aprendido}

Recordemos que el propósito de esta fase se relaciona con clarificar, profundizar y esquematizar las distintas ideas relacionadas con los textos leídos. La siguiente figura recoge las tareas desplegadas durante esta fase.

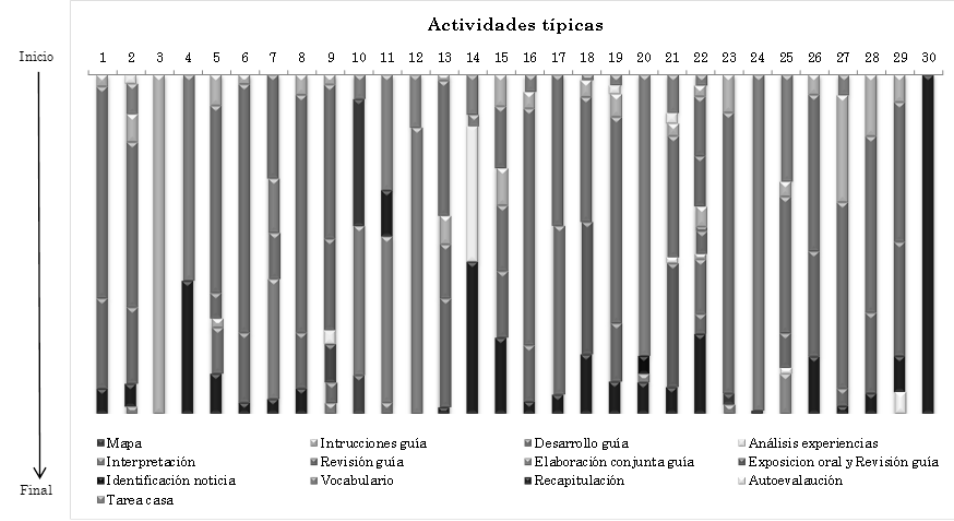

FIGURA 3 - MAPA DE EPISODIOS MOMENTO DESPUÉS DE LA LECTURA

En la figura se observa una gran variabilidad para abordar este momento. Así de los 22 tipos de episodios o tareas identificadas se incluyeron 13 de ellos en esta fase. De estos, cinco tipos de episodios fueron más recurrentes, los episodios de recapitulación que habitualmente cerraban la actividad mediante un resumen o síntesis de lo trabajado; los episodios de interpretación en los que se indagaba sobre la lectura; los episodios de instrucciones de guía de trabajo destinados a entregar orientaciones generales para responder la guía; los episodios de desarrollo de guía de trabajo que implicaban una resolución individual 
o en grupos de las preguntas, supervisado en la mayoría de los casos por los profesores en los puestos de trabajo de los estudiantes; y los episodios de revisión de guía. Dentro de los menos frecuentes se pueden mencionar los episodios de autoevaluación, mapa conceptual y análisis de experiencia.

Una de las estrategias didácticas más recurrente en esta muestra fue la articulación de distintos episodios orientados en su conjunto a realizar las guías de trabajo, que como hemos señalado anteriormente, son recursos didácticos elaborados por los profesores que buscarían clarificar y/o profundizar la comprensión lograda por los estudiantes. Dichas configuraciones se organizaron secuencialmente a partir de tres episodios distintos, instrucciones de la guía de trabajo, desarrollo de la guía de trabajo y revisión de la guía de trabajo. Cada uno de ellos suponía patrones distintos de interacción entre los profesores y los estudiantes, sin embargo, en su conjunto implicaban pasos estratégicos para realizar la tarea de profundizar en los contenidos tratados durante la lectura. En relación a su presencia, se observó que aun cuando un número importante de los profesores utilizó estas guías de trabajo, solo un porcentaje de las configuraciones fueron desplegadas en su totalidad, es decir, articulando los tres tipos de episodios. Para el resto de las actividades típicas, la manera de enfrentar esta fase consistió en desplegar episodios únicos dirigidos a interpretar o revisar vocabulario, o elaborar mapas conceptuales.

Recapitulando los hallazgos de esta fase, se pudo identificar que la mayoría de los profesores organizó esta fase en función de la utilización de recursos didácticos, sin embargo en la práctica no se observó la consecución de acciones que permitiera una continuidad de las mismas mediante la entrega de instrucciones, desarrollo y revisión de las guías de trabajo. Por otra parte, aquellas tareas orientadas a organizar las ideas esquemáticamente o propiciar la creatividad fueron menos frecuentes.

\section{Discusión y conclusiones}

Retomando el objetivo central orientado en describir la configuración de las actividades típicas de lectura colectiva, se puede precisar, en primer lugar, que fue posible identificar los tres momentos de la lectura (CLARK; GRAVES, 2005) en todas ellas. No obstante, cada una de ellas incluyó distintas tareas, particularmente en las fases durante y después de la lectura.

Al respecto, la detección de episodios de planificación y conocimientos previos permitiría plantear que todas las ATAs corresponderían a un patrón 
complejo guiado por planes (SÁNCHEZ; GARCÍA; ROSALES, 2010), es decir, en todos los procesos de enseñanza-aprendizaje existiría un propósito explícito de aquello que los estudiantes deben aprender o lograr. No obstante, se debe precisar que la muestra corresponde a evidencia audiovisual de la evaluación docente $\mathrm{y}$, en este sentido, los profesores reconocen ciertos principios que deben guiar la organización de la enseñanza (CHILE, 2007, 2008), por lo que la inclusión de estas tareas podría obedecer más a ese conocimiento, que al manejo de los principios que debieran guiar la organización de la enseñanza de la comprensión de textos (CLARK; GRAVES, 2005). Además de esto, se identificaron dos regularidades vinculadas con los momentos durante la lectura y después de la lectura. Estas regularidades consistían en ciertos episodios que se agrupaban para lograr un propósito común. Así, se detectaron tres configuraciones de episodios en la fase durante la lectura, que permitían ir supervisando la acción mediante la interpretación de lo leído, clarificar el significado de palabras o revisar la conexión entre lo leído y lo indagado en guías de trabajo. La otra configuración fue desplegada en la fase después de la lectura, y se constituía de tres episodios orientados a la realización de las tareas. En otras palabras, una de las estrategias o acciones características de la muestra fue la conjugación de dos o más tareas orientadas a una meta común.

Desde otra perspectiva y retomando el principio de andamiaje planteado por el modelo de SRE de Graves y Graves (2003), si bien fue posible observar la inclusión de las distintas fases de la lectura y la incorporación de distintas tareas orientadas a dar soporte o ayuda al proceso comprensivo de los estudiantes, se detectaron diferencias en las formas en cómo finalmente estas estrategias se desplegaron en las aulas. Así, por ejemplo, se asume la importancia de apoyar la comprensión de los estudiantes en la fase durante la lectura, a través de acciones que permitan supervisar dichas comprensiones, no obstante, sólo la mitad de los profesores incluyeron estrategias para ello, intercalando fragmentos de lectura y de interpretación de la misma, implicando ello que los estudiantes que participaron en el resto no recibieron el mismo apoyo.

De igual modo, la mayoría de los profesores planificó la profundización y el trabajo de comprensión, mediante la utilización de recursos didácticos elaborados con anterioridad, denominadas aquí guías de trabajo. Sin embargo, no en todas las actividades típicas en las que se identificó esta estrategia se pudo observar una consecución de la acción, es decir, una organización, secuenciación y temporalidad de esta que permitiera apoyar a los estudiantes en la clarificación de las preguntas que debían resolver o en la comprobación de las respuestas elaboradas por ellos.

Por último, aun cuando en todas las actividades típicas fue posible identificar un número importante de tareas que debieran desplegarse durante 
el proceso comprensivo, una de las características de la muestra fue la escasa inclusión de tareas vinculadas al desarrollo o potenciación de la creatividad de los estudiantes, que permitieran vincular las ideas del texto con sus experiencias personales. Asimismo, sólo fue posible detectar dos episodios destinados a la elaboración de mapas conceptuales, estrategia que requiere un mayor nivel de análisis en la organización y clasificación de la información.

En este sentido, la escasa inclusión de tareas que permiten establecer un puente entre lo aprendido en la lectura con su realidad (análisis de experiencias), u organizar sus ideas de acuerdo a sus propias comprensiones (mapas conceptuales), podría ser una evidencia de la necesidad de que los profesores refuercen su trabajo en esta área.

\section{REFERENCIAS}

APPLEBEE, A.; LANGER, J.; NYSTRAND, M.; GAMORAN, A. Discussion-based approaches to developing understanding: Classroom instruction and student performance in middle and high school English. American Educational Research Journal, v. 40, n. 3, p. 685-730, 2003.

CHILE. Ministerio de Educación Chileno. Jornada de Planificación de la Enseñanza. Nivel de Educación Básica. Santiago, Chile: Coordinación Nacional Enseñanza Básica, 2007.

. Jornada de Planificación de la Enseñanza. Nivel de Educación Básica. Santiago, Chile: Coordinación Nacional Enseñanza Básica, 2008.

. Reglamento sobre Evaluación Docente. Santiago: Chile: Ministerio de Educación, República de Chile, 2004.

CLARK, K. F.; GRAVES, M. F. Scaffolding students' comprehension of text. The Reading Teacher, v. 58, n. 6, p. 570-580, 2005.

DANIELS, H. Vigotsky y la Pedagogía. Barcelona: Paidós, 2003. (Temas de educación). FLICK, U. Introducción a la investigación cualitativa. Madrid: Editorial Morata, 2012.

GRAVES, M. F.; GRAVES, B. B. Scaffolding reading experiences: Designs for student success. Norwood, MA: Christopher-Gordon, 2003.

GUBA, G. Criteria for assessing the trustworthiness or naturalistic inquieres. Educational Communication and Technology, v. 29, n. 2, p. 75-91, 1981. 
LEMKE, J. Aprender a hablar ciencia. Lenguaje, aprendizaje y valores. Barcelona: Paidós, 1997. (Temas de educación).

LIANG, L.; PETERSON, C.; GRAVES, M. Investigating two approaches to fostering children's comprehension of literature. Reading Psychology, v. 26, n. 4-5, p. 387-400, 2005.

NYSTRAND, M. Research on the role of classroom discourse as it affects reading comprehension. Research in the Teaching of English, v. 40, n. 4, p. 392-412, 2006.

RUIZ, J. I. Metodología de la investigación cualitativa. Bilbao: Deusto. 2012.

2012.

. Metodología de la investigación cualitativa. España: Universidad de Deusto,

SÁNCHEZ, E.; GARCÍA, R.; CASTELLANO, N.; DE SIXTE, R.; BUSTOS, A. Qué, cómo y quién: tres dimensiones para analizar la práctica educativa. Cultura y Educación: Revista de teoría, investigación y práctica, v. 20, n. 1, p. 95-11.8, $2008 \mathrm{a}$.

SÁNCHEZ, E.; GARCÍA, R.; ROSALES, J. La lectura en el aula: qué se hace, qué se debe hacer y qué se puede hacer. Barcelona: Grao, 2010.

SÁNCHEZ, E.; GARCÍA, R.; ROSALES, J.; DE SIXTE, R.; CASTELLANO, N. Elementos para analizar la interacción entre estudiantes y profesores: ¿qué ocurre cuando se consideran diferentes dimensiones y diferentes unidades de análisis? Revista de Educación, España, n. 346, p. 105-104, mayo/ago. 2008b.

STODOLSKY, S. La importancia del contenido en la enseñanza. Actividades en las clases de matemáticas y ciencias sociales. Barcelona: Paidós, 1991. (Temas de educación).

WELLS, G. Indagación dialógica. Hacia una teoría y una práctica socioculturales de la educación. Barcelona: Paidós, 2001. (Temas de educación).

Texto recebido em 12 de julho de 2014 Texto aprovado em 26 de setembro de 2014. 